\title{
Mini-Review
}

Theme: Pediatric Drug Development and Dosage Form Design

Guest Editors: Maren Preis and Jörg Breitkreutz.

\section{Personalized Medicine in Pediatrics: The Clinical Potential of Orodispersible Films}

\author{
J. Carolina Visser, ${ }^{1,3}$ Herman J. Woerdenbag, ${ }^{1}$ Lidwien M. Hanff, ${ }^{2}$ and Henderik W. Frijlink ${ }^{1}$
}

Received 14 January 2016; accepted 15 March 2016; published online 4 April 2016

\begin{abstract}
Children frequently receive medicines that are designed for adults. The dose of commercially available products is adapted, mostly based on the child's bodyweight, thereby neglecting differences in pharmacokinetic and pharmacodynamics parameters. If commercial products are unsuitable for administration to children or are unavailable, extemporaneous pharmacy preparations are a good alternative. For this particular population, orodispersible films (ODFs) can be a highly attractive dosage form for the oral administration of drugs. ODFs are relatively easy to prepare in a hospital setting, create dose flexibility, and may suit an individual approach, especially for patients having difficulties in swallowing tablets or being fluid restricted. In this article, various aspects related to pharmacy preparations, clinical application, and preparation of ODFs for pediatric patients are highlighted and discussed.
\end{abstract}

KEYWORDS: Clinical possibilities; Extemporaneous pharmacy preparations; Orodispersible films.

\section{INTRODUCTION}

Personalized medicine is becoming increasingly important in contemporary pharmacotherapeutics. Children ranging from preterm newborns and neonates via infants and toddlers to adolescents form an important target group. Frequently, they receive a medicine that is designed for adults, a practice called "unlicensed" or "off label use," i.e., not licensed for that particular purpose. The administered dose for children is often based on body weight, length, and gender only, thereby neglecting clinically important differences in pharmacokinetics (absorption, metabolism, and elimination of the drug) and pharmacodynamics (changes in receptor expression). Nowadays, serious attempts are being made to develop medicines, formulations, and dosage forms that are adapted to the specific needs of children. Several scientific guidelines have been released by the Food and Drug Administration (FDA) and the European Medicines Agency (EMA) concerning the development of medicines for children. But there are still hurdles to overcome: the safety of the excipients, the palatability, and appropriate taste masking need to be taken into account [1-11].

\footnotetext{
${ }^{1}$ Department of Pharmaceutical Technology and Biopharmacy, University of Groningen, Antonius Deusinglaan 1, 9713 AV, Groningen, The Netherlands.

${ }^{2}$ Erasmus MC-Sophia Children's Hospital Rotterdam, Wytemaweg 80, 3015 CN, Rotterdam, The Netherlands.

${ }^{3}$ To whom correspondence should be addressed. (e-mail: j.c.visser@rug.nl; )
}

It has been recognized that children are unable to or have difficulties with swallowing tablets or capsules. Even up to the age of 18 years, children may have difficulties swallowing tablets of $15 \mathrm{~mm}$ diameter [12]. With adequate training techniques, children can be taught to swallow smaller tablets. (Un)coated minitablets with a size of 2-3 $\mathrm{mm}$ appear to be well tolerated in trained children at the age of 6 months- 6 years [13-16]. However, caution is needed if coated minitablets are administered to children below 1 year of age because of incidences of cough during ingestion [15] and since children tend to chew on them [13]. To circumvent swallowing problems, tablets are often cut into halves or even quarters, leading to inaccurate dosing or an altered absorption [2, 17]. Tablets may be crushed and capsules may be opened, only if they are immediate release products. Most tablets or capsules with modified release properties are absolutely unsuitable for this purpose. Subsequently, the powder can be mixed with a spoonful of liquid or food. However, parents or caretakers may unintentionally mix the powder with a substance that may alter stability or influence absorption [18].

Liquid formulations are a suitable alternative to solid dosage forms. They are easy to administer and create dose flexibility. However, there are some limitations related to liquid dosage forms. Frequently, such dosage forms are too strong for children. On the other hand, if the available liquid dosage forms are not strong enough, it is not always possible to achieve the required dosage. Especially in children suffering from disorders that require fluid restriction, this is a relevant issue. Furthermore, there is a considerable risk of under- or overdosing errors. To overcome these problems and to increase the safety for the patient, e.g., reducing chocking risk due to particle inhalation by giving chewing 
tablets, and reducing dosage errors by offering a formulation with an adapted dose, orodispersible drug formulations such as orodispersible films (ODFs) are an attractive new and innovative administration form for this particular target group [9, 19].

If commercially available products are unsuitable or unavailable, extemporaneous preparations can fulfill the patient's individual need. An important precondition, however, is that extemporaneous manufacturing is performed under circumstances that guarantee a good product quality. In hospital pharmacies or large manufacturing pharmacies, such preconditions can be achieved and thus pediatrics preparations can be manufactured there for the individual patient [3]. ODFs can be both commercially prepared and extemporaneously on a small scale in a hospital setting as pharmacy preparations. According to the European Pharmacopoeia Eighth Edition (Ph. Eur. 8th Edition), orodispersible films are single- or multilayer sheets of suitable materials, to be placed in the mouth where they disperse rapidly [20]. ODFs are easy to administer and require almost no intake of water. ODFs can be cut precisely in any desired dosage strength with a pair of scissors. Hence, they may suit the individual approach needed in pediatrics [21, 22].

The focus of this paper is on pharmacy preparations, clinical possibilities, and preparation of extemporaneously prepared ODFs to be applied in pediatrics.

\section{PHARMACY PREPARATIONS CREATE FLEXIBILITY}

According to the European Pharmacopoeia, pharmacy preparations include extemporaneous preparations made for an individual patient or for a small patient population which are supplied immediately after preparation, as well as stock preparations which are made in advance and stored until requested [23]. In view of this definition, pharmacy preparations are very suitable to overcome the problem related to a lack of commercially available medicines for pediatric patients and the need of tailor-made, personalized medicines for children. Pharmacy preparations can be compounded from raw materials, made by modification or manipulation of commercially available licensed dosage forms or prepared prior to use [2,24]. Figure 1 gives an overview of the main problems related to the manipulation of commercially available oral dosage forms and provides some good alternatives.

In The Netherlands, the Royal Dutch Pharmacist Association (KNMP), the professional organization for pharmacists, has developed standardized procedures covering various dosage forms and basic manufacturing processes. They are available online (but no open access) for Dutch pharmacists and updated on a regular basis. Many of these standardized procedures are incorporated and discussed in the book Practical Pharmaceutics, an international guideline for the preparation, care, and use of medicinal products [24]. A Dutch formulary is available with standardized formulations for smaller-scale pharmacy preparations for which no licensed alternative is available. These formulations yield good and reliable products if prepared according to the general procedures, thereby safeguarding general quality. The use of these products is considered on a benefit to risk evaluation for specific patient categories such as pediatric patients. Oral (liquid and solid), dermal, oropharyngeal, ocular, nasal, rectal, and parenteral preparations are included in the monographs as well as comments with relevant background information for each individual formulation. The standardized pharmacy preparations can be applied to make individual prescriptions or for the preparation of small to

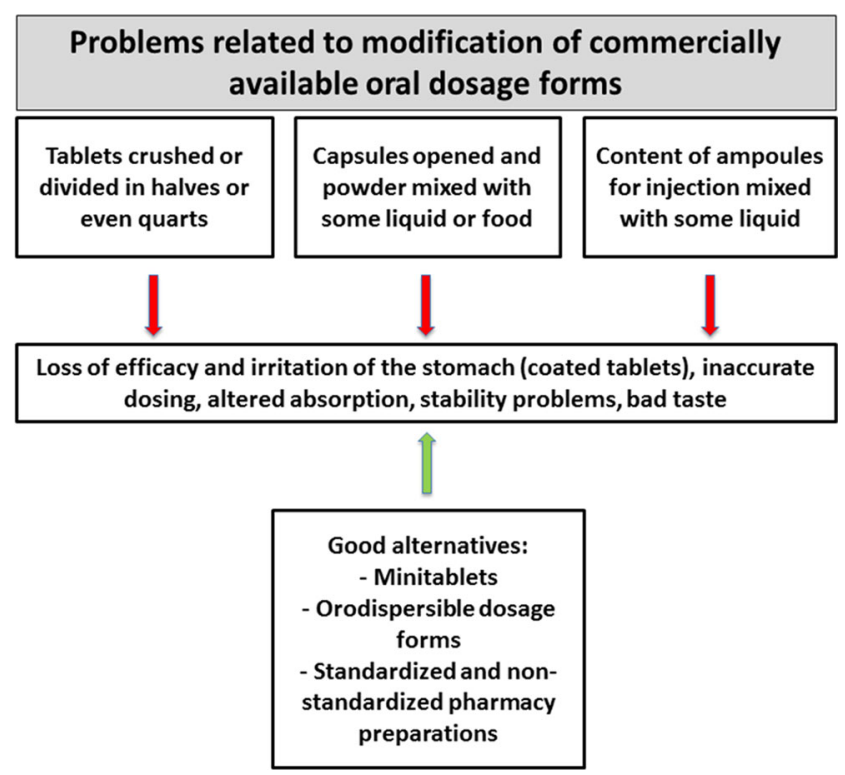

Fig. 1. Main problems related to the modification of commercially available oral dosage forms and good alternatives

medium stocks. All standardized preparations have been thoroughly validated and yield high quality and reproducible products [25]. Current initiatives by the European Directorate for the Quality of Medicines and Healthcare aim to harmonize the specific class of pediatric pharmacy preparations [26]. Up till now, ODF preparations for pediatrics are not available as standardized formulations. However, research in this field is ongoing. Good quality ODFs containing low-dose enalapril maleate, prednisolone, or diazepam have been successfully prepared on lab scale which could be suitable as standardized formulations [21]. Nonstandardized pharmacy preparations are used for single or at most for a handful of patients. The effectiveness, safety, and quality of the product should always be evaluated before it is prepared. Choosing a non-standardized pharmacy preparation means that no formulation is readily available. The design and the technological production process have to be drafted from scratch and will result in a product that complies with a minimum set of requirements regarding composition, quality, stability, and biopharmaceutical performance. Furthermore, the product's practical applicability and ease of use as well as the safety for the patient and the compounder must be taken into account. Also, the balance between the risk of non-availability of a medicine and the risk of well-documented quality of the product should be considered. The pharmacist takes the final responsibility in this respect and has an advising role to the prescribing physician [25].

\section{GMP CONDITIONS}

The production of medicines should be performed under conditions that guarantee the quality of the product and its chemical, physical, and microbiological stability. In the pharmaceutical industry worldwide, strict good manufacturing practice (GMP) conditions are applied [27, 28]. In a hospital or compounding pharmacy setting where pharmacy preparations are produced in small batches, a full industrial GMP process is not feasible. Furthermore, specific requirements unique for a hospital setting are not addressed in the industrial GMP guidelines [25]. To guide good practices for 
the preparation of medicinal products in healthcare establishments, the Pharmaceutical Inspection Convention in 2008 published the PIC/S guidelines which are being increasingly used as a reference for pharmacy preparations including aseptic handling in pharmacies in Europe [24, 29]. In The Netherlands, the industrial GMP guidelines have been adapted to fit to the hospital environment to the so-called GMP-Z, established by the Dutch Hospital Pharmacists Association (NVZA) in 1996 and revised in 2009-2011 [24, 30]. The GMP-Z encompasses guidelines to ensure that good quality pharmacy preparations are manufactured in the hospital under controlled conditions. The GMP-Z also covers preparation processes that are specific for a hospital pharmacy but not described in the industrial GMP [25]. The hospital specific preparation processes are described in four additional chapters: assessment of the requested pharmacy preparation and the quality of the product design; tailor-made preparations; aseptic handling; guidelines how to work with hazardous (starting) materials and products [30].

Tailor-made preparations are defined in the GMP-Z guideline as preparations intended for one or a few patients which are supplied immediately and not kept in stock. Such preparations can be compounded from validated standardized preparation instructions or from non-standardized preparation instructions. ODFs as extemporaneous pharmacy preparations can be covered by the tailor-made preparations as defined in this GMP-Z guideline. Validation, in-process control, and end-control of tailor-made preparations differ from those for stock preparations. For that reason, all specified instructions need to be followed precisely. Besides, personnel should be qualified and trained adequately and continuously $[24,30]$.

\section{PRESCRIPTION ASSESSMENT}

In principle, for each pharmacy preparation, a product file needs to be established. In a product file, all documents regarding the pharmacy preparation are collected, e.g., pharmacotherapeutic rationale, instructions regarding the preparation, operating- and analytical instructions, and stability and user information [24]. An important step in the product file is the assessment of the therapeutic rationale which entails the decision whether there is a specific clinical need for a particular pharmacy preparation. In other words, does the patient benefit from it in terms of pharmacotherapeutic outcome and is the patient not exposed to too much risk. In pediatric formulation development of new dosage forms such as extemporaneously prepared ODFs, this is an inevitable step.

Considerations that need to be taken into account are: is a licensed and commercial product available? Does the pharmacy preparation have a unique therapeutic value for the particular patient? Does it improve patient-friendliness and hence better compliance? Is the pharmacy preparation safe for the patient? [22, 26, 31] A risk assessment is needed if an extemporaneous pharmacy preparation is required where the benefit of the patient and the possible risk involved with the preparation should be considered in relation to the appropriate quality level. For each pharmacy preparation, the quality needs to be assured and preparation methods should be uniform, reproducible, and well documented.
Documentation is therefore an important part of the quality system related to pharmacy preparations [24]. Figure 2 gives an overview of the steps involved in a prescription assessment.

\section{CLINICAL POSSIBILITIES FOR THE APPLICATION OF ODFS TO PEDIATRICS}

Drugs are administered orally to children not only in the outpatient setting [32], but also in the hospital. Here, a substantial proportion of drugs is administered orally even to children admitted to neonatal and pediatric intensive care units. The use of oral drugs, as opposed to intravenously administered drugs, has the advantage of less risk of adverse effects including infection, less costs, and easier administration without the need for an inconvenient intravenous access. In infants and neonates, a liquid formulation is the most widely used oral dosage form because of the advantage of dose flexibility combined with a lower risk on choking, as opposed to solid oral dosage forms.

However, for many drugs commonly used in pediatrics, a suitable drug formulation or dosage strength is not commercially available. In the past decade, progress has been made following American and European legislation, to stimulate pharmaceutical companies to perform studies in children, but a large proportion of older, generically available drugs, will not be included in these programs $[33,34]$. For many drugs commonly used in pediatrics, such as antiepileptic drugs (phenytoin, phenobarbital), diuretics (hydrochlorothiazide, furosemide and spironolactone), ACE inhibitors (enalapril), and calcium channel antagonists (nifedipine, amlodipine), no formulation is commercially available, which is suitable for use in neonates or small children. For these drugs, oral liquids have been developed as extemporaneous pharmacy preparations. However, taste or stability issues or solubility difficulties hamper the applicability of some liquids. As an example, hydrochlorothiazide is often used as a long-term treatment in neonates for the treatment of bronchopulmonary dysplasia, a

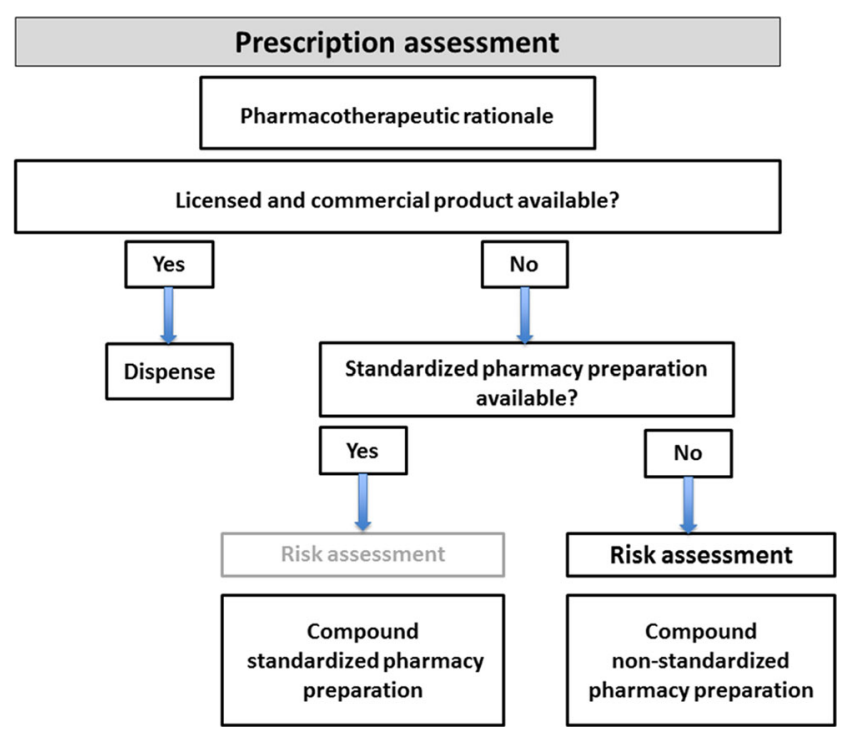

Fig. 2. Prescription assessment 
chronic lung disorder. Hydrochlorothiazide is soluble up to $0.6 \mathrm{mg} / \mathrm{ml}$ in an aqueous environment. Higher concentrations can only be prepared as a suspension instead of a clear solution, leading to a potential risk on dosage errors when not homogenized properly prior to administration. Therefore, a solution of $0.5 \mathrm{mg} / \mathrm{ml}$ hydrochlorothiazide is most often prepared.

However, the current pediatric dosage of $1-2 \mathrm{mg} / \mathrm{kg}$ orally twice daily results in an undesired high volume intake when using the $0.5-\mathrm{mg} / \mathrm{ml}$ concentration. For these children, the use of a drug formulation, which combines an easy administration, dose flexibility with low volume fluid intake might be beneficial. As such, the use of hydrochlorothiazide in an ODF might be an important improvement in drug formulation. ODFs are easy to administer and need almost no intake of water. Once administered, an ODF sticks to the tongue or palatal and cannot be spit out thereby improving patient compliance. Different ODF formulations have already been developed which are suitable as a starting point for further research, e.g., ODFs containing caffeine, dimenhydrinate, enalapril maleate, prednisolone, diazepam, or ibuprofen [21, 35-37]. The used APIs should be appropriately taste masked and non-irritable. Due to the limited drug load per ODF, multiple dosing might be necessary which might be considered as a disadvantage $[8$, $9,22,31]$.

In other clinical conditions where volume restriction is important, ODFs may gain in place in the therapeutic arsenal. Many cardiovascular drugs are administered to patients in which the fluid balance is rigidly monitored and carefully balanced, because of a comprised cardiac output. The limited volume associated with the use of ODFs may well be an advantage in its clinical use, as opposed to larger volumes of liquid.

\section{DEVELOPMENT AND TECHNICAL ASPECTS OF AN ODF FORMULATION}

ODFs can be relatively easily prepared on a small scale using the solvent casting method. A particular ODF consists of an active pharmaceutical ingredient (API), a film-forming polymer, or a combination of film-forming polymers, a plasticizer, and various excipients such as flavors, colors, fillers, saliva-stimulating agents, and, although not often used, a preservative [22, 38]. All used excipients should be appropriate and safe for the target group, especially for children $[8,31]$. In brief, the preparation of an ODF using the solvent casting method is as follows. The API and all excipients are combined in an appropriate solvent. The API is preferably dissolved in water but can also be dissolved in a co-solvent, dissolved as an API-cyclodextrin complex or finely dispersed as a suspension [21, 35-38]. After a clear solution or homogeneous suspension has been obtained, the casting solution is casted with a desired casting height varying from 500 to $2000 \mu \mathrm{m}$ onto a release liner. The release liner is fixed by vacuum suction on a film applicator. The casting solution is dried on the film applicator at low temperature and subsequently cut into the desired size [21, 22]. Size and drug content of the initial casting solution will determine the final dose. Besides the solvent casting method, printing of an API onto a plain ODF is an upcoming manufacturing method which could become very interesting for the small-scale production of ODFs [39, 40].

Before ODFs can be prepared as small-scale extemporaneous pharmacy preparations for clinical use, the formulation has to be developed, tested, and optimized on a lab scale. The casting solution must be free from air bubbles, should contain no lumps of polymers or aggregates of API or excipients, and should possess a viscosity making it suitable for casting. The prepared ODFs should be easy to remove from the release liner, not stick to the fingers during handling but do stick well to the tongue or palatal, to prevent chocking in the pediatric patient group, where they disintegrate fast. They meet the requirements of uniformity of mass and uniformity of content, possess suitable mechanical properties (do not break during handling), and show physical, chemical, and microbiological stability during storage [21, 22]. A current restriction is the limited drug load per ODF; therefore, only relatively potent APIs can be used. Up to now, a few studies are available concerning the acceptance of ODFs. However, it is assumed that the acceptability is high in children on the condition that the ODFs have acceptable taste [22].

From various pre-formulation studies, it appeared that different APIs and dosages may require a specific composition of the casting solution. The APIs may influence the viscosity of the casting solution to such an extent that the solution becomes unsuitable for casting and ODF production. This was most evident for enalapril maleate. For poorly water-soluble APIs such as diazepam, a co-solvent is needed. The use of a co-solvent may also negatively influence the viscosity of the casting solution. Besides, a test on residual solvent is needed as oral intake of the solvent is harmful to the target group. Typically, such problems may require a different composition of the casting solution [21].

The quality by design (QbD) approach was shown to be a useful tool to optimize ODF formulations [41]. QbD is a systemic approach to improve the control over the quality of the production process and includes the establishment of a quality target profile [41, 42]. Subsequently, the optimized formulation can be used for the repetitive production of ODFs in a hospital setting on a small scale for clinical use. In such setting, the ODFs need to be prepared under GMP conditions and rigid quality control should guarantee a continuously good product. Besides the aforementioned requirements, an important requirement in the clinic is that the ODFs are sufficiently resistant to damage that may be caused by handling. ODFs need special air-tight packaging to maintain integrity and stability. The suitability of aluminum sachets, foil, paper, or plastic pouches as packaging material needs to be evaluated carefully $[22,38]$.

\section{PERSPECTIVE}

Pharmacy preparations such as ODFs create more dose flexibility than achievable with commercially available products for adults, provide good patient acceptance, and hence increase patient compliance [22]. ODFs can be cut into separate smaller pieces for dose adjustment, which is important in pediatrics [21]. ODFs are easy to administer and require almost no intake of water. This makes ODFs a suitable dosage form for patients who have difficulties swallowing large amounts of fluid, are unable to swallow (mini)tablets, are non-cooperative, and are prone to 
spitting out drugs or suffer from disease requiring restricted fluid intake, e.g., oedema or heart failure. This shows the perspectives for the administration of ODFs to neonates and pediatrics. The majority of the API will be released in the oral cavity and subsequently swallowed resulting in a pharmacokinetic profile compared to an oral solution [22]. However, the first-pass metabolism can be partly circumvented if absorption via the oral mucosa is promoted. This may improve bioavailability which entails a risk of overdosing if the ODFs are not administered correctly. On the other hand, an improved bioavailability may reduce the dose needed and contribute to a reduction of side effects [22, 43]. ODFs are also convenient portable dosage forms. This is an advantage compared to brittle and fragile orodispersible tablets and liquid dosage forms that demand a special package for transportation or are only available in large bottles [43].

Up to now, there are no standard formulations for the preparation of ODFs available in the Dutch or in any other national formulary. However, research in this field is ongoing and promising.

Open Access This article is distributed under the terms of the Creative Commons Attribution 4.0 International License (http://creativecommons.org/licenses/by/4.0/), which permits unrestricted use, distribution, and reproduction in any medium, provided you give appropriate credit to the original author(s) and the source, provide a link to the Creative Commons license, and indicate if changes were made.

\section{REFERENCES}

1. Ivanovska V, Rademaker CMA, van Dijk L, Mantel-Teeuwisse A. Pediatric drug formulations: a review of challenges and progress. Pediatrics. 2014;134(2):361-72.

2. Standing JF, Tuleu C. Paediatric formulations-getting to the heart of the problem. Int J Pharm. 2005;300:56-66.

3. 't Jong GW, Vulto AG, de Hoog M, Schimmel KJM, Tibboel D, van den Anker JN. A survey of the use of off-label and unlicensed drugs in a Dutch children's hospital. Pediatrics. 2001;108(5):1089-93.

4. Strickley RG, Iwata Q, Wu S, Dahl TC. Pediatric drugs-a review of commercially available oral formulations. J Pharm Sci. 2008;97(5):1731-74.

5. Turner MA, Catapano M, Giaquinto C, On behalf of GRIP (Global Research in Paediatrics). Paediatric drug development: the impact of evolving regulations. Adv Drug Deliv Rev. 2014;736:2-13.

6. Nahata MC, Allen Jr LV. Extemporaneous drug formulations. Clin Ther. 2008;30(11):2112-9.

7. Florence AT, Attwood D. Physicochemical principles of pharmacy. In: Manufacture, formulation and clinical use. 6th Edition. Chapter 10. Paediatric and geriatric formulations. London: Pharmaceutical Press; 2016. p. 427-41.

8. Walsch J, Cram A, Woertz K, Breitkreutz J, Winzenburg G, Turner R, et al. Playing hide and seek with poorly tasting paediatric medicines: do not forget the excipients. Adv Drug Deliv. 2014;73:14-23.

9. Preis M. Orally disintegrating films and mini-tablets-innovative dosage forms of choice for pediatric use. AAPS PharmSciTech. 2015;16(2):234-41.

10. European Medicines Agency (EMA). Scientific guidelines: Paediatrics. http://www.ema.europa.eu/ema/index.jsp? curl= pages/regulation/general/general_content_000404.jsp\&mid= WC0b01ac0580926186. Accessed 28 Feb 2016.

11. United States Food and Drug Administration (FDA). Pediatric product development. http://www.fda.gov/Drugs/Development
ApprovalProcess/DevelopmentResources/ucm049867.htm. Accessed 28 Feb 2016.

12. Guideline on pharmaceutical development of medicines for paediatric use, 2013, European Medicines Agency, via http:// www.ema.europa.eu/docs/en_GB/document_library/Scientific_ guideline/2013/07/WC500147002.pdf. Accessed 05 Jan 2016.

13. Kluk A, Sznitowska M, Brandt A, Sznurkowska K, Plata-Nazar $\mathrm{K}$, Mysliwiec $\mathrm{M}$, et al. Can preschool-aged children swallow several minitablets at a time? Results from a clinical pilot study. Int J Pharm. 2015;485:1-6.

14. Spomer N, Klingman V, Stoltenberg I, Lerch C, Meissner T, Breitkreutz J. Acceptance of uncoated mini-tablets in young children: results from a perspective exploratory cross-over study. Arch Dis Child. 2012;97(3):283-6.

15. Klingman V, Spomer N, Lerch C, Stiltenberg I, Frömke C, Bosse $\mathrm{HM}$, et al. Favorable acceptance of mini-tablets compared with syrup: a randomized controlled trial in infants and preschool children. J Pediatr. 2013;163:1728-32.

16. Klingman V, Seitz A, Meissner T, Breitkreutz J, Moeltner A, Bosse HM. Acceptability of uncoated mini-tablets in neonates-a randomized controlled trial. J Pediatr. 2015;167(4):893-6.

17. Van Santen E, Barends DM, Frijlink HW. Breaking of scored tablets: a review. Eur J Pharm Biopharm. 2002;53:139-45.

18. Terry Ernest (UK), Athens, European Paediatrics Formulation Initiative Conference 2014, (17-18 Sept 2014).

19. Slavkova M, Breitkreutz J. Orodispersible drug formulations in children and elderly. Eur J Pharm Sci. 2015;75:2-9.

20. Oromucosal preparations. European pharmacopoeia. 8th ed. Strasbourg: Council of Europe; 1807.

21. Visser JC, Woerdenbag HJ, Crediet S, Gerrits E, Lesschen MA, Hinrichs WLJ, et al. Orodispersible films in individualized pharmacotherapy: the development of a formulation for pharmacy preparation. Int J Pharm. 2015;478:15563.

22. Krampe R, Visser JC, Frijlink HW, Breitkreutz J, Woerdenbag HJ, Preis M. Oromucosal film preparations: points to consider for patient centricity and manufacturing processes. Expert Opin Drug Deliv. 2015. doi:10.1517/17425247.2016.1118048.

23. Pharmaceutical preparations (2619). European Pharmacopoeia, 8th edition, Council of Europe, Strasbourg

24. Bouwman-Boer Y, Fenton-May V, Le Brun P. Practical pharmaceutics, an international guideline for the preparation, care and use of medicinal products. Switzerland: Springer international publishing; 2015.

25. Woerdenbag HJ, Visser JC, Frijlink HW. Pharmacy preparations, customized quality in Dutch clinical care. In: The University of Medicine and Pharmacy at Ho Chi Minh City, Vietnam, ed. Proceeding Pharma Indochina VIII. ASEAN Pharmacy Integration for Development. 4-5 December 2013. Nhà Xu t B n Y H c (Medical Publishing House). 2013; 19-22.

26. European Directorate for the Quality of Medicine via www.edqm.eu. Accessed 05 Jan 2016.

27. The rules governing medicinal products in the European Union. EudraLex volume 4: guidelines for good manufacturing practices for medicinal products for human and veterinary use, version V27, February 2013. Website: http://ec.europa.eu/health/documents/eudralex/vol-4/index_en.htm.

28. Forty-fifth report of the WHO Expert Committee on Specifications for Pharmaceutical Preparations. (WHO Technical Report Series, No. 961), WHO, Geneva, 2011. Website: www.who.int/ medicines/publications/pharmprep/en/index.html.

29. The Pharmaceutical Inspection Convention and Pharmaceutical Inspection Co-operation Scheme. www.picscheme.org. Accessed 03 Mar 2016.

30. GPM guidelines for hospital pharmacies in The Netherlands. Website: http://nvza.nl/voor-professionals/gmp/ . Accessed 04 Mar 2016.

31. Zajicek A, Fossler MJ, Barrett JS, Worthington JH, Ternik R, Charkoftaki G, et al. A report from the pediatric formulations task force: perspectives on the state of child-friendly oral dosage forms. AAPS J. 2013;15(4):1072-81.

32. Schirm E, Tobi H, de Vries TW, Choonara I, De Jong-van den Berg LTW. Lack of appropriate formulations of medicines for children in the community. Acta Paediatr. 2003;92:1486-9. 
33. European Medicines Agency (EMA). Paediatric Regulation. http:// www.ema.europa.eu/ema/index.jsp?curl=pages/regulation/ document_listing/document_listing_000068.jsp. Accessed 01 Mar 2016.

34. Unites States Food and Drug Administration (FDA). Pediatric Research Equity Act (PREA). http://www.fda.gov/Drugs/ DevelopmentApprovalProcess/DevelopmentResources/ ucm049867.htm. Accessed 01 Mar 2016.

35. Garsuch V, Breitkreutz J. Comparative investigations on different polymers for the manufacturing of fast-dissolving oral films. J Pharm Pharmacol. 2010;62:539-45.

36. Preis M, Pein M, Breitkreutz J. Development of a taste-masked orodipersible film containing dimenhydrinate. Pharmaceutics. 2012;4(4):551-62.

37. Woertz C, Kleinebudde P. Development of orodispersible polymer films containing poorly water soluble active pharmaceutical ingredients with focus on different drug loadings and storage stability. Int J Pharm. 2015;493(1-2):134-45.

38. Hoffmann EM, Breitenbach A, Breitkreutz J. Advances in orodispersible films for drug delivery. Expert Opin Drug Deliv. 2011;8:299-316.
39. Preis M, Breitkreutz J, Sandler N. Perspective: concepts of printing technologies for oral film formulations. Int J Pharm. 2015;494:578-84.

40. Janßen EM, Schliephacke R, Breitenbach A, et al. Drugprinting by flexographic printing technology-a new manufacturing process for orodispersible films. Int J Pharm. 2013;441:818-25.

41. Visser JC, Dohmen WMC, Hinrichs WLJ, Breitkreutz J, Frijlink HW, Woerdenbag HJ. Quality by design approach for optimizing the formulation and physical properties of extemporaneously prepared orodispersible films. Int J Pharm. 2015;485:70-6.

42. IHC Q8 International Conference of Harmonization, 2008. ICH Harmonized Tripartite Guideline: Q8 (R1) Pharmaceutical Development. Website: http://www.ich.org/products/ guidelines/quality/article/quality-guidelines.html. Accessed 04 Mar 2016.

43. Borges AF, Silva C, Coelho JF, Simões S. Oral film: current status and future perspectives I-galenical development and quality attributes. J Control Release. 2015;206:1-9. 